\title{
Assistive Pen to Improve Quality Writing of Hand Tremor with Proportional-Control
}

\author{
Z. M. Yusop, M. Z. Md. Zain, M. Hussein, A. R Musa, and I. Ishak
}

\begin{abstract}
Patient who is suffering hand tremor may find difficulty in performing handwriting task. Responding to this problem, the assistive pen is developed by examining the effect of proposing the Proportional-control in counter the hand tremor affect to the quality of handwriting. Experiments were conducted on an assistive pen placed on the experimental rig that will resemble actual hand tremor writing. The Linear Voice Actuator (LVCA) is used as an anti-tremor device. Based on real-time experiments result, proposed proportional controller shows an enormous potential on an assistive pen in counter tremor movement.
\end{abstract}

Index Terms-Assistive pen, proportional controller, linear voice coil actuator.

\section{INTRODUCTION}

Tremor disease is an involuntary or rhythmic uncontrollable movement that may occur to one or more body parts but it may clearly visible to hand rather than other body parts. A neurological disorder is another symptom of tremor that is suffered by patients, also known as, Parkinson's disease (PD). The patients who suffer from PD face the difficulty of controlling some of their body parts from shaking or oscillating, and it is commonly visible in the hands and head.

Tremor patients will face difficulties, especially when performing daily tasks such as holding and writing. Handwriting capabilities become worse when the tremor is severe. The oscillation of hand during writing may affect the quality of handwriting. Therefore, this research has been conducted by presenting biomechanical loading approach without surgical interventions or ingestion of drugs. Recently, the application of biomechanical loading has shown to be a technique that is able to suppress the uncontrollable movement caused by tremor on the human body. Micron like intelligent active handheld microsurgical instrument has become a great interest in the field of microsurgery, helping a surgeon perform better Ophthalmological microsurgery procedure due to physiological tremor occurring during operations [1]. The device will sense the undesired motion

Manuscript received March 19, 2014; revised August 10, 2014. This work was supported in part by Universiti Teknologi Malaysia (UTM) and Malaysia's Ministry of Higher Education (MOHE) for providing continuous support, especially in term of funding through GUP (Vot:06J31) and PRGS (Vot:4L616).

Z. M. Yusop, M. Z. Md. Zarhamdy, M. Hussein, A. R Musa, and I. Ishak are with the Department of Applied Mechanics and Design, Faculty of Mechanical Engineering, Universiti Teknologi Malaysia, 81310 Johor, Malysia (email: zul_12521@yahoo.com, zarhamdy@fkm.utm.my, mohamed@fkm.utm.my). and counter it with an equal but opposite deflection of the instrument's tip. MARo2 [2] is a meal-assist robot developed by E. Ohara et al. (2009) where the human-machine interface makes it possible for the person with a tremor to manipulate the supporting robot without causing operability to deteriorate and without hazards due to improper operation. This non-invasive method will measure the unwanted movement due to the tremor using appropriate sensor and suppress the movement with smart actuator.

This study is intended to develop such a writing pen that can counter the hand tremor during writing, and to study the performance of the implementation of Proportional-Integral-Derivative (PID) controller to the assistive pen. The Proportional controller from PID control was selected due to extensive use in the industry field [3]. The heuristic method is employed to tune the Proportional parameter to achieve the desired target which is 'zero' tremor. The sensitivity analysis for each tuning value of the parameter is proper discussed in this paper. The implementation of this control technique shows the invention of this assistive pen is created a great potential to reduce the legibility of handwriting quality among people with hand tremor.

\section{SYSTEM DESCRIPTION}

The research was conducted by presenting experimental rig set up that intended to locate an assistive pen. The experimental rig is developed which able to emulate the actual handwriting tremor behavior. The crucial part of this experimental rig development is the actuator selection. The actuator must have enough force and fast time response to generate tremor when the tremor signal is applied. To meet the criteria, bidirectional Linear Voice Coil Actuator (LVCA) with model NCC 05-11-011-1X from h2w is selected with the specifications of peak force of $14.7 \mathrm{~N}$, which is enough to support vibration load and the low operating voltage, $\pm 3 \mathrm{~V}$. In order to apply the tremor signal to the experimental rig, a male participant (38-year-old) has participated in data measurement. This participant is diagnosed to have Parkinson disease (PD) according to clinical criteria and have difficulties to perform daily hand task especially handwriting. To take a measurement, the participant was asked to track several words on the paper by using a common pen that was mounted with accelerometer (MMA7361LCR1).

As shown in Fig. 1, the back end of LVCA is attached to the aluminium plate to act as a fixed part of the system and the front end of LVCA is connected to the movable part that will hold an assistive pen. When the tremor signal is applied to LVCA, the moving parts will create a movement to leftward and rightward from its original position. The two linear 
bearings are installed between fixed and moving parts and hence minimal the friction and provide smooth horizontal movement. Meanwhile, the DC motor acts as spur to create a straight line to the pen point by turning the linear screw thread and the movement is perpendicular to the direction of tremor.

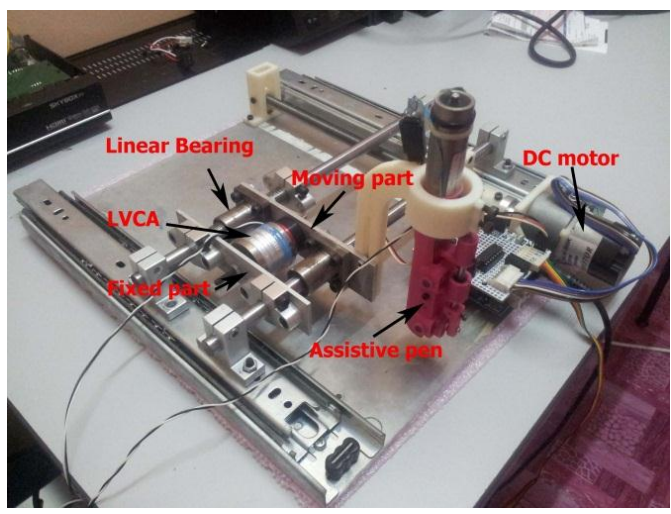

Fig. 1. Experiment-rig setup.

For the assistive pen it is designed to be suited with the tremor patient fingers grip and similarly have functioned with regular pen but some features are added to control the tremor. Once again, LVCA as the actuator is selected as the actuator.

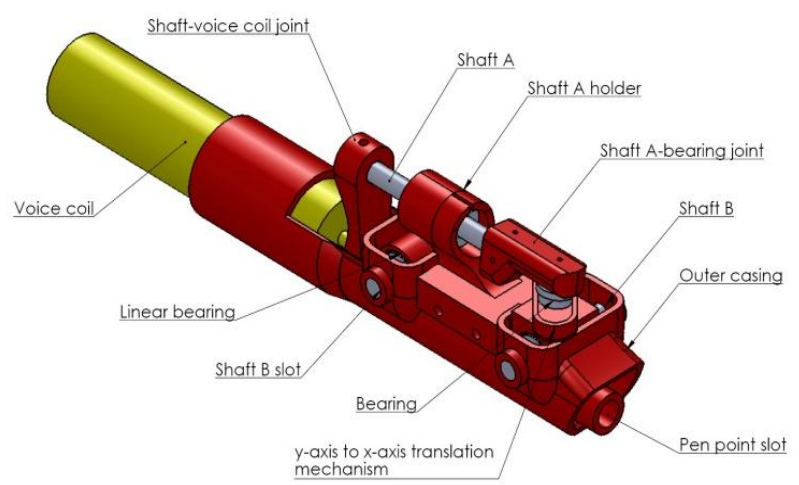

Fig. 2. Assistive pen.

As shown in Fig. 2, LVCA is mounted inside the back end of the pen. The moving LVCA shaft is connected to the joints and bearings to move the pen point in rightward and leftward. To activate the actuator, a feedback acceleration signal from a miniature accelerometer (MMA7361LCR1) board with dimension of $5 \mathrm{~mm}(\mathrm{~W}) \times 11 \mathrm{~mm}(\mathrm{~L}) \times 1.75 \mathrm{~mm}(\mathrm{~T})$ is embedded inside the pen. The signals detected by the accelerometer were then sent to the computer through NI-DAQ card (PCI-6259) to do further control system analysis.

Before acceleration signal can be further analyzed, the noise interferes during the measurement need to be filtered. The second-order Butterworth filter is proposed with band-pass type selection between the frequency range of $0.175-15 \mathrm{~Hz}$. This frequency range is selected properly to conserve the frequencies interest of a tremor which is in a range of $3-12 \mathrm{~Hz}$ [4] and at the same time eliminate the noise. The signal processing and control part were done with LabVIEW graphical user interface software package and for faster online control, a low level programming environment is necessary to reduce computational buffering [5].

\section{CONTROL SCHEME}

\section{A. PID Controller}

A Proportional-Integral-Derivative controller (PID) or also known as "three-term" controller [6] is a generic control close loop feedback extensively used in industrial control system. In this experimental study, the Proportional controller which a part of PID controller is used to control the speed response and the stroke of the LVCA. The parameter of proportional gain is tuned with heuristic approach. Generally, in the PID controller, there are three-term control structure consisting of proportional action, integral action and derivative action. By tuning the three parameters in the PID controller algorithm, the controller can provide controlled action designed for specific process requirements. The proportional gain $(K p)$, Integral gain $(K i)$, and Derivative gain $(K d)$ terms are summed to calculate the output of PID controller. The equation of PID algorithm is as below. The $S P$ is ideal value of output and $S V$ the measured value.

$$
K c(t)=K p \cdot e(t)+K i \cdot \int_{0}^{t} e(\tau) \cdot e \tau+K d \cdot \frac{d}{d t} e(t)
$$

Since the $K p$ gain is only considered and the $K i$ and $K d$ are left to be zero, rearrange (1);

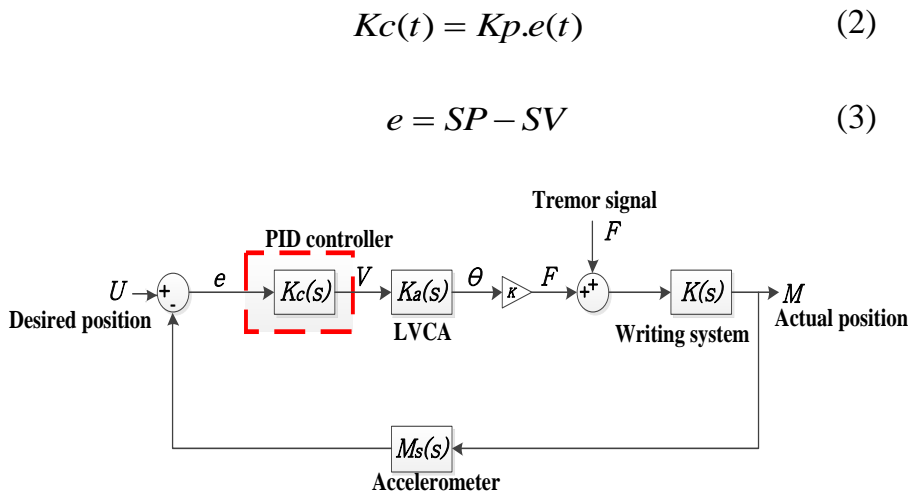

Fig. 3. the schematic diagram of propose controller.

\section{RESULTS}

This topic discusses the results obtained from performing analysis with using m-file code which is part of the MATLAB software environment. The power spectral density (PSD) is used to evaluate the performances of assistive pen based on reduction of coherence amplitude and display the signal in the time and frequency domains.

The Fig. 4, present the comparison of the proposed proportional gain controller as the gain varies with respect to the acceleration behavior. The result was plotted in 10 seconds time series. The result indicates that the $\mathrm{P}$ controller gain of 0.42 gives better attenuation control of tremor compared with other values. As shown in Fig. 4(a), the acceleration response is plotted in time domain and based on the result, it is not easy to estimate the tremor attenuation causes of uneven signal response. This matter can be solved by plotting the acceleration response in the frequency domain as shown in Fig. 4(b). Based on the figure, frequency peak of gain 0.42 successes to suppress the tremor peak by $56.57 \%$, 
followed by 0.40 with $55.46 \%, 0.38$ with $52.48 \%$ and 0.35 with $44.04 \%$. For a gain of 0.00 means there is no control is applied and act as a reference frequency for the other gains to attenuate the peak frequency. This figure shows the proposed controller is able to attenuate the frequency peak at the same location which is at $8.667 \mathrm{~Hz}$ and except for gain 0.40 which slightly strayed from the other with peak frequency occurred at $8.728 \mathrm{~Hz}$. This problem probably causes the effect of noise interference during experimental is conducted, the LVCA by itself generates the magnetic field when activated and hence more or less affect the accelerometer reading inside the pen which is placed close to it. However, the range coherence frequency is still in the accepted frequency range of actual hand tremor.

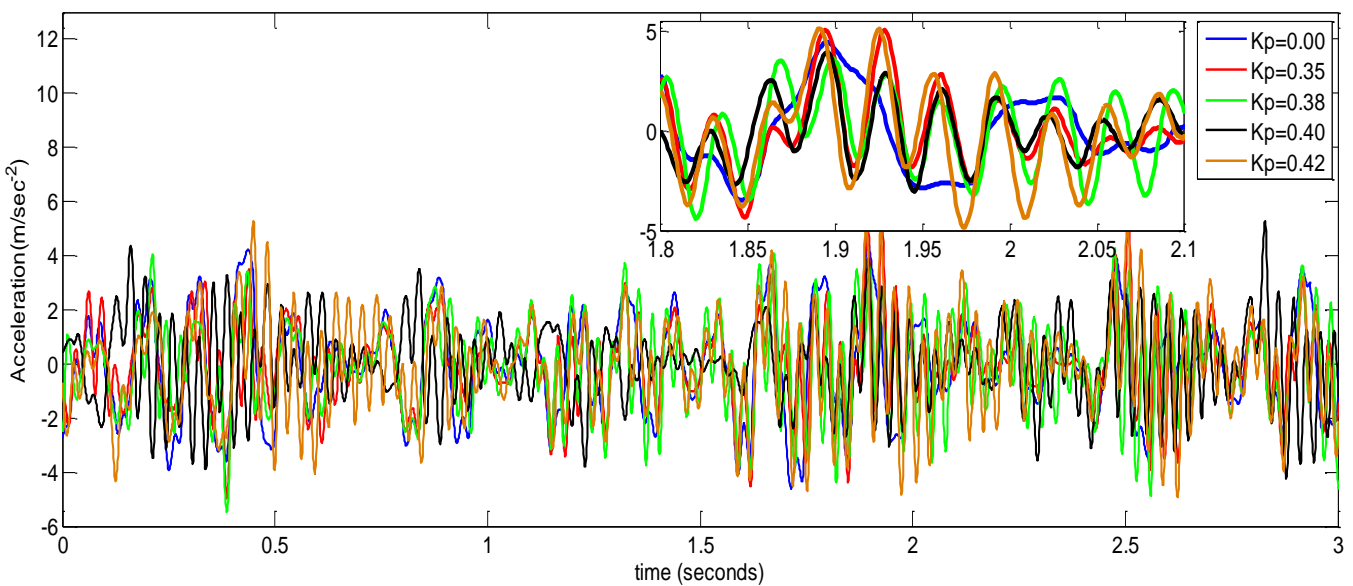

(a)

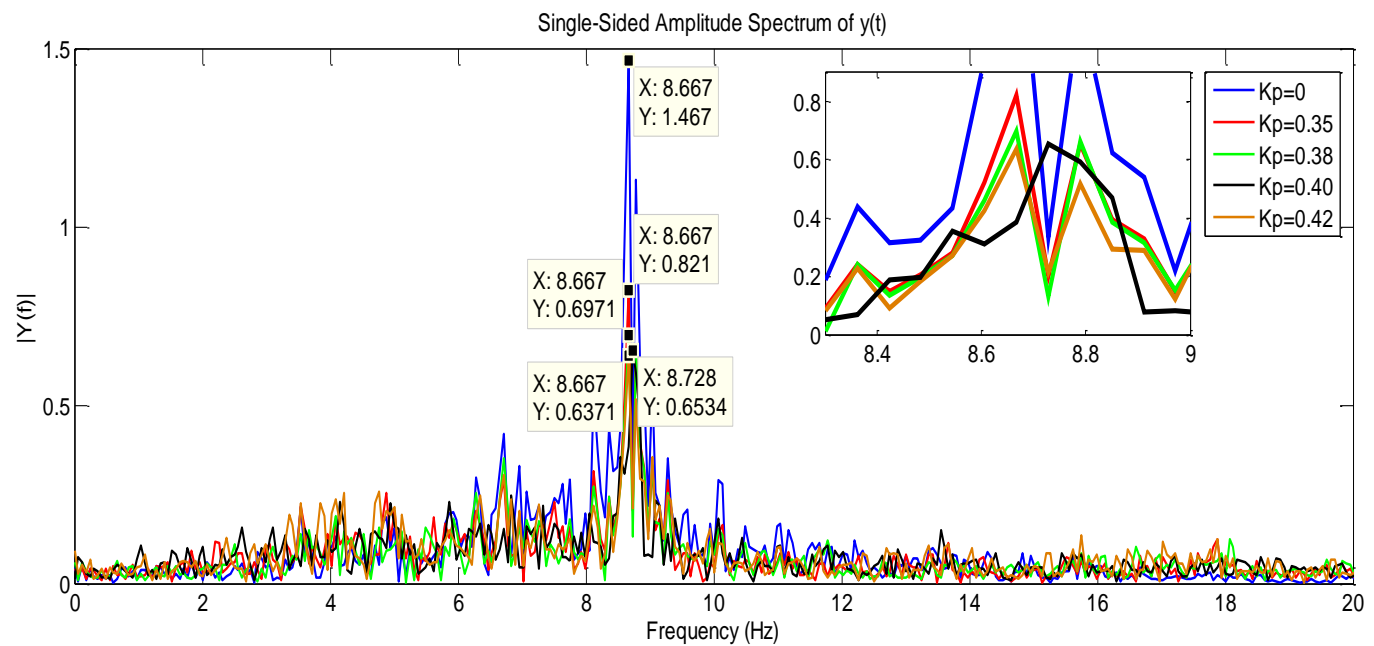

(b)

Fig. 4. Analysis of proposed controller on acceleration and PSD: (a) Time domain and (b) Frequency domain.

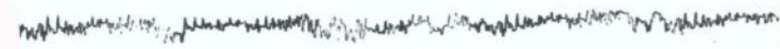

(a)

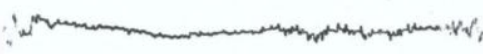

(b)

Fig. 5. Handwriting quality of an assistive device: (a) gain 0.00 (b) gain 0.42 .

Fig. 5, depicts the performance of an assistive pen in attempt to writing a straight line as desired path of the experimental objective. Fig. 5(a) shows the performance of a pen when injecting original tremor signal without applying any gain control. The tremor occurred almost at the straight line path with an average of $4.2 \mathrm{~mm}$ is measured. However, Fig. 5(b) shows the attenuation of tremor when a gain of 0.42 is applied. The attenuation processing is not uniform due to the random signal input and from the measurement the average of reduction is $2 \mathrm{~mm}$, roughly.

\section{DISCUSSION AND CONCLUSION}

This experiment works with real-time implementation using proposed controller is presented. The selection of LVCA provided a good performance to the system. A miniature accelerometer provided an advantage to embed inside the small scale assistive pen and also act as a feedback signal to the system. From the results analysis, the proposed proportional gain controller provided a good result in counter hand tremor movement during handwriting. This preliminary result of proposed controller may assist further implementation of advance control schemes.

\section{ACKNOWLEDGMENT}

The authors would like to thank Universiti Teknologi 
Malaysia (UTM) and Malaysia's Ministry of Higher Education (MOHE) for providing continuous support, especially in term of funding through GUP (Vot:06J31) and PRGS (Vot:4L616).

\section{REFERENCES}

[1] W. T. Ang, C. N. Riviere, and P. K. Khosla, "Design and Implementation of Active Error Cancelling in Hand-held Microsurgical Instrument," in Proc. IEEE International Conference on Intelligent Robots and Systems, Maui, Hawaii, USA, 2001, pp. 1106-1111.

[2] E. Ohara, "Tremor Suppresion Control of Meal-Assist Robot with Adaptive Filter," in Proc. $11^{\text {th }}$ IEEE International Conference on Rehabilitation Robotics, Kyoto Japan, 2009, pp. 498-503.

[3] Y. Yan, W. A. Klop, M. Molennar, and P. Nijdam, "Tuning a PID controller: particle swarm optimization versus genetic algorithm," 2010.

[4] P. David, M. D. Charles, J. Gregory, B. S. Esper, L. Thomas, M. D. Davis, J. Robert, M. D. Maciunas, and M. D. Robertson, "Classification of Tremor and update Treatment," American Family Physician, vol. 59, no. 6, pp. 1565-1572, 1999.

[5] A. As'arry, M. Z. M. Zain, M. Mailah, and M. Hussein, "Hybrid Learning Control for Improving Suppression of Hand Tremor," Proceeding journal of Engineering in Medicine, 2013, pp. 1-10.

[6] K. H. Ang, G. Chong, and Y. Li, "PID Control System Analysis, Design, and Technology," IEEE Transaction on Control Systems Technology, 2005, vol. 13, no. 4, pp. 559-576.

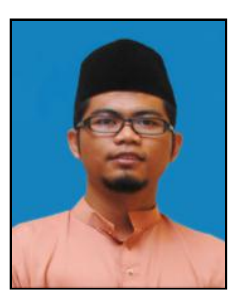

Zulkifli Mohd Yusop was born in Johor, Malaysia on October $19^{\text {th }}, 1987$. He obtained his bachelor degree in electrical control and instrumentation from the Universiti Teknologi Malaysia, Malaysia, in 2010. His major field of study is control and mechatronics. He is currently a doctor of philosophy student in mechanical engineering at the Universiti Teknologi Malaysia, Malaysia. His research interests include vibration control, system modeling, control system, simulation and human vibration.

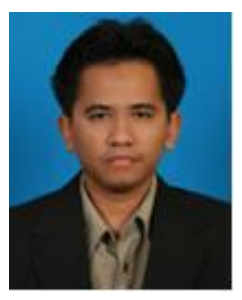

Mohd Zarhamdy Md. Zain was born in Perlis, Malaysia on August $8^{\text {th }}, 1977$. He obtained his bachelor degree in mechanical engineering from the Universiti Teknologi Malaysia, Malaysia, in 1999 before acquiring a master of science and philosophy of doctor qualification from the University of Sheffield, UK in 2002 and 2006 respectively both in control engineering. His major field of study is flexible robot control and mechatronics. $\mathrm{He}$ is currently an associated professor at the Universiti Teknologi Malaysia, Malaysia and his research interests include iterative learning control, applied mechatronics, system modeling and simulation.

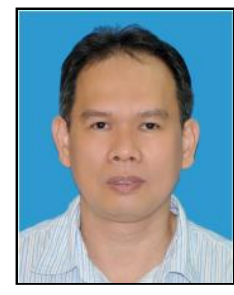

Mohamed Hussein graduated from UTM with a bachelor degree in mechanical engineering in 1987 and acquired a master of science degree in advanced manufacturing system and technology from University of Liverpool, UK in 1992. He completed his $\mathrm{PhD}$ degree at the De Montfort University, UK (Leicester) in 2008 in the field of machine control and mechatronics. He is currently an associated professor and the head of Department of Applied Mechanics, Faculty of Mechanical Engineering, UTM. His research interests in mechatronics include machine control, robotics, real-time virtual control and industrial automation.

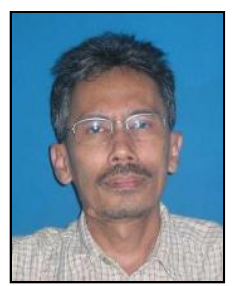

Abdul Rahman Musa was born in Perak, Malaysia. He obtained his bachelor degree in mechanical engineering with honours at Sunderland Polytechnic, United Kingdom in 1987. His major field of study is system dynamics and control. He is currently an assistant lecturer, and also pursuing his study for doctor degree of philosophy in Faculty of Mechanical Engineering at the Universiti Teknologi Malaysia, Malaysia. His research interests include mechanical design, system dynamics and control.

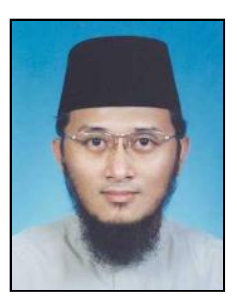

Idris Ishak was born in Kuala Lumpur, Malaysia on April $27^{\text {th }}, 1961$. He obtained his bachelor degree in design ( industrial design) from the Mara Institue of The Technology Shah Alam, Malaysia, in 1984. His completed his master degree in industrial design (engineering) at Manchester Polytechnic, Manchester, England, 1988. He is currently a senior lecturer in Faculty of Mechanical Engineering at the Universiti Teknologi Malaysia, Malaysia. His research interests include Industrial machine and design including automotive field. 